\title{
Circular Hough Transform for Iris localization
}

\author{
Noure ddine Che rabit ${ }^{*}$, Fatma Zohra Chelali, Amar Dje radi
}

Speech communication and signal processing laboratory, Faculty of Electronic en gineering and computer science University of Science and Technology Houari Boumedienne (USTHB), Box n :32 El Alia, 16111, Algiers, Algeria

\begin{abstract}
Th is article presents a robust method for detecting iris features in frontal face images based on circular Hough transform. The software of the application is based on detecting the circles surrounding the exterior iris pattern from a set of facial images in different color spaces. The circular Hough transform is used for this purpose. First an edge detection technique is used for finding the edges in the input image. After that the characteristic points of circles are determined, after which the pattern of the iris is extracted. Good results are obtained in different color spaces.
\end{abstract}

Keywords Hough Transform, Iris Detection, Face Recognition, Face Detection

\section{Introduction}

Identification of people is getting more and more importance in the increasing network society[1]. Biometrics is the branch of science in which human beings are identified with their behavioral or physical characteristics. Physical characteristics include face, finger, iris, retina, hand geometry; palm print etc. where as behavioral characteristics include signature, gait, voice...etc. In all of these characteristics, iris recognition is gaining more attention because the iris of every person is unique and it never changes during a person's lifetime[2].

Generalized iris recognition consists of image acquisition, iris segmentation and localization (preprocessing), feature extraction and feature comparison (matching). Biometric based personal identification using iris requires accurate iris localization from an eye image[3].

Several researchers have implemented various methods for segmentation and localising the iris. John Daugman[5] has proposed one of the most practical and robust methodologies, constituting the basis of many functioning systems. He used integro-differential operator to find both the iris inner and outer boundaries for iris segmentation. Wildes[4] proposed a gradient based binary edge map construction followed by circular Hough transform for iris segmentation[2].

Almost all methods stated are based on the assumptions that centre of iris (Outer Boundary) and Pupil (Inner boundary) is same and iris is perfectly circular in shapes, which are practically incorrect. Therefore, the iris segmentation and localization from an acquired image leads to the loss of texture data near to pupil and/or outer iris boundary.

* Corresponding author:

nourchera@yahoo.fr (Noureddine Cherabit)

Published online at http://journal.sapub.org/scit

Copyright (C) 2012 Scientific \& Academic Publishing. All Rights Reserved
The effect is more serious when the iris is occluded[2].

In recent years, various methods of iris detection for gaze detection, applied to man-machine interfaces have been proposed[1, 2, 3, 4]. However, most studies did not report the relationship between the accuracy rate of the iris detection and the directions of the face and the eyes. Actually, the shape of facial parts is not only different depending on the person, but also dynamically changes depending on the movement of the head, facial expressions, and so on. Also, when a person faces down, the eye regions tend to be dark. In this case, it is difficult to detect iris in the face. However, most studies are based on research of the eye region to detect iris. Where, these methods extract the eye regions before detecting irises.

An authentication system for iris recognition is reputed to be the most accurate among all biometric methods because of its acceptance, reliability and accuracy. Ophthalmologists originally proposed that the iris of the eye might be used as a kind of optical fingerprint for personal identification. Their proposal was based on clinical results that every iris is unique and it remains almost unchanged in clinical photographs. The human iris begins to form during the third month of gestation and is complete by the eighth month, though pigmentation continues into the first year after birth. It has been discovered that every iris is unique since two people (even two identical twins) have uncorrelated iris patterns [5], and yet stable throughout the human life. It is suggested in recent years that the human irises might be as distinct as fingerprint for different individuals, leading to the idea that iris patterns may be used as unique identification features[11].

Research in the area of iris recognition has been receiving considerable attention and a number of techniques and algorithms have been proposed over the last few years.

A number of algorithms have been developed for iris localization. One of them is based on the Hough transform. An iris segmentation algorithm based on the circular Hough 
transform is applied in $[6,7,8]$. At first, the canny edge detection algorithm is applied. The eye image is represented using edges by applying two thresholds to bring out the transition from pupil to iris and from iris to sclera. Then circular Hough transform is applied to detect the inner and outer boundaries of the iris. The circular Hough transform is emp loyed to deduce the radius and centre coordinates of the pupil and iris regions. In this operation, the radius intervals are defined for inner and outer circles. Starting from the upper left corner of iris the circular Hough transform is applied. This algorithm is used for each inner and outer circle separately. The votes are calculated in the Hough space for the parameters of circles passing through each edge point. Here some circle parameters may be found. The parameters that have maximum value are corresponded to the centre coordinates [6].

After determining centre coordinates, the radius $r$ of the inner circle is determined. The same procedure is applied for the outer circle to determine its centre coordinates and radius. Using determined inner and outer radiuses the iris region is detected. The application of the Hough transform needs long time to locate the boundaries of the iris [6].

The aim of this work is to realize an application which detects the iris pattern from an eye image. The software of the application is based on detecting the circles surrounding the exterior iris pattern. Figure 1 shows an overview of the proposed method. Our work concerns the iris detection by the geometric model "Hough transform" using the facial images of the "Fei-Fei Li" database[9], 396 images of different subjects with 24 frames per individual are used.

The paper is presented as follows: section 2 presents the state of the art for iris localization and recognition; Section 3 describes the circular Hough transform for known and unknown radius. Section 4 presents the algorithm applied for iris detection; results obtained for facial images are presented in section 5. Conclusion is drawn in section 5.

\section{State of the art for Iris Localization}

Methods such as the Integro-differential, Hough transfor $\mathrm{m}$ and active contour models are well known techniques in use for iris localisation. These methods are described below including their strengths and weaknesses [11].

\subsection{Daug man_s Integro-Differential Operator}

In order to localise an iris, Daugman proposed an Integro-differential operator method. It assumes that the pupil and limbus are circular contours and operate as a circular edge detector. Detecting the upper and lower eyelids is also carried out using the Integro-differential operator by adjusting the contour search fro $\mathrm{m}$ circular to a designed arcuate shape[11]. The Integro-differential operato $\mathrm{r}$ is defined as follows:

$$
\operatorname{Max}\left(r, x_{0}, y_{O}\right) G_{\sigma}(r) * \frac{\partial}{\partial r_{\left(r, x_{0}, y_{0}\right)}} \int_{2 \pi r} \frac{I(x, y)}{2 \pi} d s \mid(1)
$$

The operator pixel-wise searches throughout the raw input image, $\mathrm{I}(\mathrm{x}, \mathrm{y})$, and obtains the blurred partial derivative of the integral over normalised circular contours in different radii. The pupil and limbus boundaries are expected to maximize the contour integral derivative, where the intensity values over the circular borders would make a sudden change. $G_{\sigma}(r)$ is a smoothing function controlled by $\sigma$ that smoothes the image intensity for a more precise search[11].

This method can result in false detection due to noise such as strong boundaries of upper and lower eyelids since it works only on a local scale.

\subsection{Hough Transfor $m$}

The Hough Transform is an algorithm presented by Paul Hough in 1962 for the detection of features of a particular shape like lines or circles in dig italized images [18].

The classic Hough Transform is a standard algorithm for line and circle detection. It can be applied to many computer vision problems as most images contain feature boundaries which can be described by regular curves. The main advantage of the Hough transform technique is that it is tolerant to gaps in feature boundary descriptions and is relatively unaffected by image noise, unlike edge detectors[17].

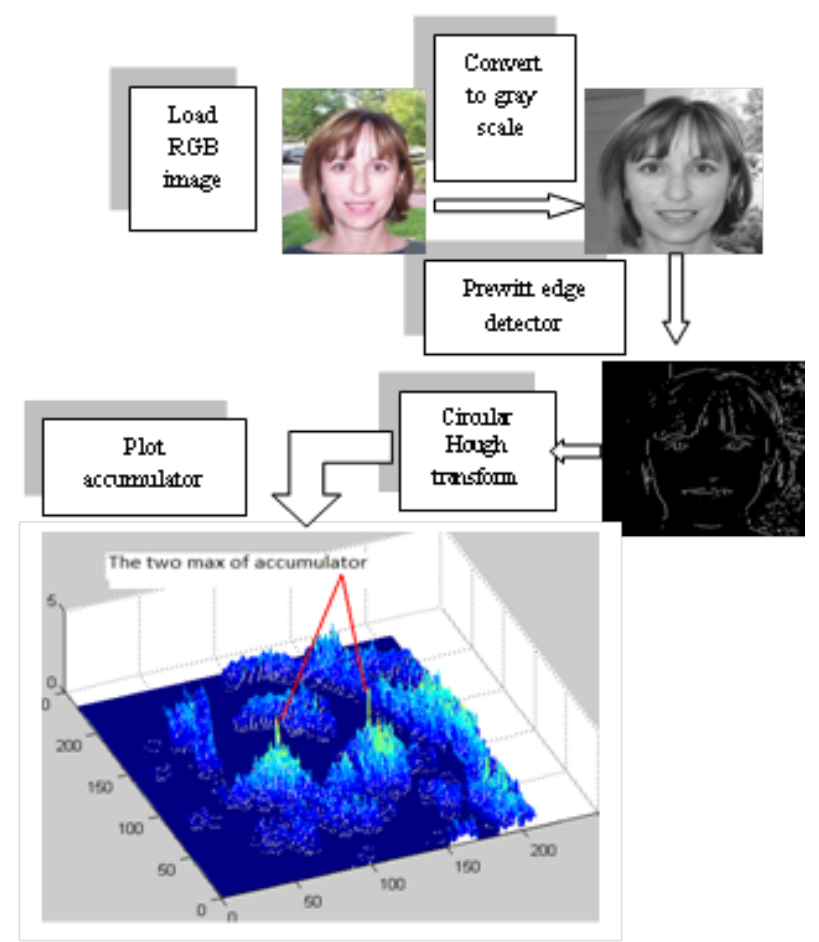

Figure 1. General overview of Iris localization 
Hough transform is a standard image analysis tool for finding curves that can be defined in a parametrical form such as lines and circles. The circular Hough transform can be employed to deduce the radius and centre coordinates of the pupil and iris regions[11].

Wildes[12], Kong and Zhang[13], Tis se et al.[14] and Ma et al.[15] have all used Hough transform to localize irises. The localization method, similar to Daugman's method, is also based on the first derivative of the image intensity. In the method proposed by Wildes, an edge map of the image is first obtained by thresholding the magnitude of the image intensity gradient:[11].

$$
|\nabla G(x, y) * I(x, y)|
$$

Where:

$$
\begin{aligned}
& \nabla \equiv\left(\frac{\partial}{\partial x}, \frac{\partial}{\partial y}\right) \text { and } \\
& G(x, y)=\frac{1}{2 \pi \sigma^{2}} e^{-\frac{\left(x-x_{0}\right)^{2}+\left(y-y_{0}\right)^{2}}{2 \sigma^{2}}}
\end{aligned}
$$

$\mathrm{G}(\mathrm{x}, \mathrm{y})$ is a Gaussian smoothing function with scaling parameter $\sigma$ selecting the proper scale of edge analys is. The edge map is then used in a voting process to maximise the defined Hough transform for the desired contour. A maximum point in the Hough space will correspond to the radius $r$ and centre coordinates $x_{c}$ and $y_{c}$ of the circle best defined by the edge points according to equation:

$$
x_{c}^{2}+y_{c}^{2}-r_{c}^{2}=0
$$

Wildes et al. and Kong and Zhang also make use of the parabolic Hough transform to detect the eyelids by approximating the upper and lower eyelids with parabolic $\operatorname{arcs}[11]$.

The Hough transform method requires the threshold values to be chosen for edge detection, and this may result in critical edge points being removed, thus resulting in failures to detect circles/arcs. In addition, Hough transform is computationally intensive due to its "brute-force" approach, and thus may not be suitable for real time applications[11].

\subsection{Discrete Circular Active Contours}

Ritter proposed an active contour model to localise iris in an image. The model detects pupil and limbus by activating and controlling the selected active contour using two defined forces: internal and external[11].

The internal forces are designed to expand the contour and keep it circular. This force model assumes that pupil and limbus are globally circular, rather than locally, to minimise the undesired deformations due to peculiar reflections and dark patches near the pupil boundary. The contour detection process of the model is based on the equilibrium of the defined internal forces with the external forces. The external forces are obtained from the grey level intensity values of the image and are designed to push the vertices inward. The movement of the contour is based on the composition of the internal and external forces over the contour vertices. Each vertex is moved between time $t$ and $(\mathrm{t}+1)$ by

$$
V_{i}(t+1)=V_{i}(t)+F_{\text {int }, i}+F_{\text {ext }, I}
$$

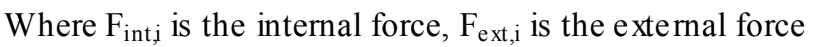
and $V_{i}$ is the position of vertex $i$.

A point interior to the pupil is located from a variance image and then a discrete circular active contour (DCAC) is created with this point as its centre. The DCAC is then moved under the influence of internal and external forces until it reaches equilibrium, and the pupil is then localised.

\section{Hough Transform Algorithm}

\subsection{Principal of Hough Transform}

In an image, a curve "in any form" can be defined by a set of points. There is generally a set of parameters that links these points by their spatial information, or coordinate information in the image space. So it means that the curve is parameterized and can be modeled by a mathematical equation that gives the relationship between the two sets (for example, the equation of a line, circle...).

Thus, the general principle of the Hough transform is a projection of the $\mathrm{N}$-dimensional image space (denoted by I and defined by its variable $\mathrm{i}=1: \mathrm{N}$ ) to a parameter space with a dimension $\mathrm{M}$ (denoted by $\mathrm{H}$ and defined by its variable $\mathrm{j}=1: \mathrm{M})$. The two spaces are related by the mathematical model $x_{i}=f\left(a_{i}\right)$. So the objective of the work is to find the parameters defining the shape to be detected.

For example, a straight line is determined by equation: $y=m x+p$, where $\mathrm{m}$ represents the slope and $\mathrm{p}$ the intercept. Then the Hough transform is given by the equation:

$$
I=\{\mathrm{x}, \mathrm{y}\} \stackrel{\mathrm{TH}}{\longrightarrow} H=\{\mathrm{m}, \mathrm{p}\}
$$

There are two kinds of Hough transform (depending on the formulation of the mathematical equation linking the two spaces):

-Transformation of 1 to $\mathrm{m}$ (one point of $\mathrm{I}$ is associated with $m$ points of $H$ ).

-Transformation of $\mathrm{m}$ to 1 (m-points of I are associated with one point of $\mathrm{H}$ ).

In practice the Hough space $\mathrm{H}$ is discretized. The result of Hough transform is stored in a table cell of two dimensions (in the case of space (m, p) named the Hough accumulator). Thus, each cell position $m_{i}, p_{i}$ has its own accumulator[10].

\subsection{Hough Transform for Circles Detection}

The Hough transform can be applied to detect the presence of a circular shape in a given image. It is used to detect any shape or to locate the iris in the face[10].

The characteristic equation of a circle of radius $r$ and center $(a, b)$ is given by: 


$$
(x-a)^{2}+(y-b)^{2}=r^{2}
$$

This circle can be described by the two following equations:

$$
\begin{aligned}
& x=a+r \cos (\theta) \\
& \mathrm{y}=\mathrm{b}+\mathrm{r} \sin (\theta)
\end{aligned}
$$

Thus, the role of the Hough transform is to search for the triplet of parameters $(a, b, r)$ which determines the points $\left(\mathrm{x}_{\mathrm{i}}, \mathrm{y}_{\mathrm{i}}\right)$. Two cases may be presented as described in Fig 2 .

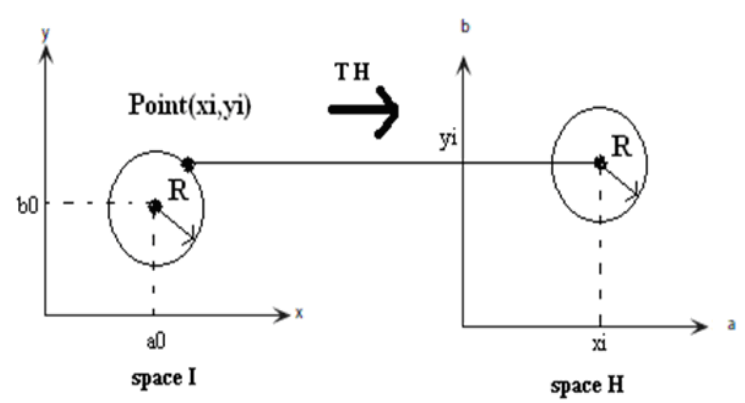

Figure 2. Transformation of a point in circle[10]

\section{- Case of known radius}

If we know the radius of the circle to be detected in the image, the parameter to search is reduced to a pair $(a, b)$ and the $\mathrm{H}$ space is 2 dimensional.

We consider a circle of radius $\mathrm{R}$ and center $\left(a_{0}, b_{0}\right)$, the transformation for each point $\left(x_{i}, y_{j}\right)$ in space I yields a circle in space $\mathrm{H}$ having a center $x_{i}, y_{j}$ and radius $\mathrm{R}$.

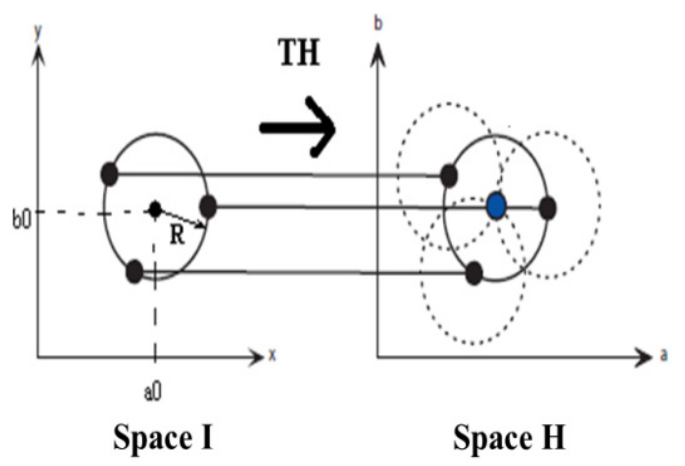

Figure 3. Representation of HT for several points in circle[10]

Similarly, we transform all points of the circle in image. The result will be more circles where their intersection is the point $\left(a_{0}, b_{0}\right)$. This point is obtained by searching the maximu m of the accumu lator.

\section{-Case of unknown radius}

In this case, the work consists to find the triplet parameters $\left(a_{0}, b_{0}, r_{0}\right)$ witch define the points of circle to find. The space will be in 3D.

For each point $\left(x_{i}, y_{i}\right)$ of the space I will match a cone in space $H$, as the radius $r$ varied from 0 to a given value (figure 4). After transforming of all points of contour in the same way, the intersection will give a spherical surface corresponding to the maxim of accumulator. The area is characterized by a center $\left(a_{0}, b_{0}\right)$ and the radius $r_{0}$ searched[10].

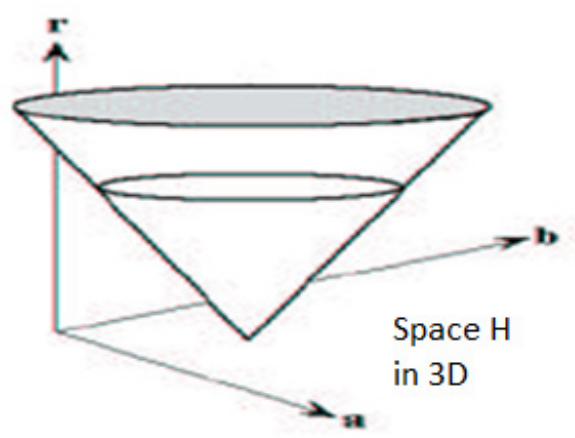

Figure 4. Transformation of a point for an unknown radius[10]

\section{Iris Detection System}

Before the Hough transform is applied to the image, there was an edge detection technique used for finding the edges in the input image. First, we convert the image into the gray scale. Then extract the essential information of contour present in the image.

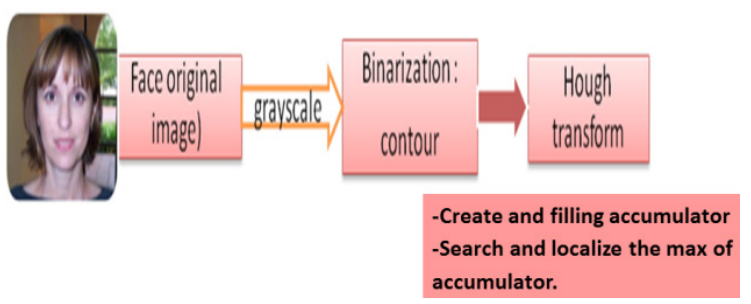

Figure 5. Different steps of treatment

For this, we choose the 'prewitt filter' to extract the contour image. After the circles are detected and drawn on the original image then, the pattern of the iris is extracted and stored. A simple overview of the corresponding steps is shown in figure 5.

The next step consists to apply our method required the following steps:

After creating an accumulator $h$ with the same dimension of the image, we extract the contour of image with the Prewitt filter. Then a model of the circle that satisfies the equation: $x^{2}+y^{2}=r^{2}$, where $\mathrm{r}$ is the radius, is created.

The second step consists of taking each circle with a radius $\mathrm{Ri}$, we do a scan of image on the contour and placing the circle on each non-zero point of this picture (contour), and increment each position of points (xi, yi) of the accumulator by one for each intersection of all point with any point of contour.

In the final step, we locate the position $\mathrm{Xi}$ and $\mathrm{Yi}$ of $\mathrm{N}$ maximum points of accumulator; $\mathrm{N}$ represents the number of radius to search.

We can summarize this work as follows:

-Read RGB Image: Converting the RGB image into grayscale level. 
- Extract the contour by the Prewitt filter (b inary image).

- Introduce the variation range of the iris radius (in pixels)

- Creation of models and centralize of circles.

- Search and locate the position of the maximums in accumulator.

- Extract the positions X0i, Y0i for each radius ri.

- Display the image and the circles found satisfying equation $\left(x-x_{0 i}\right)^{2}+\left(y-y_{0 i}\right)^{2}=r^{2}$

The research of parameters that define the circles has been limited with centers $\left(x_{0}, y_{0}\right)$ and radius $r$. To get a good results, we give each radius corresponds to an interval of iris rays.

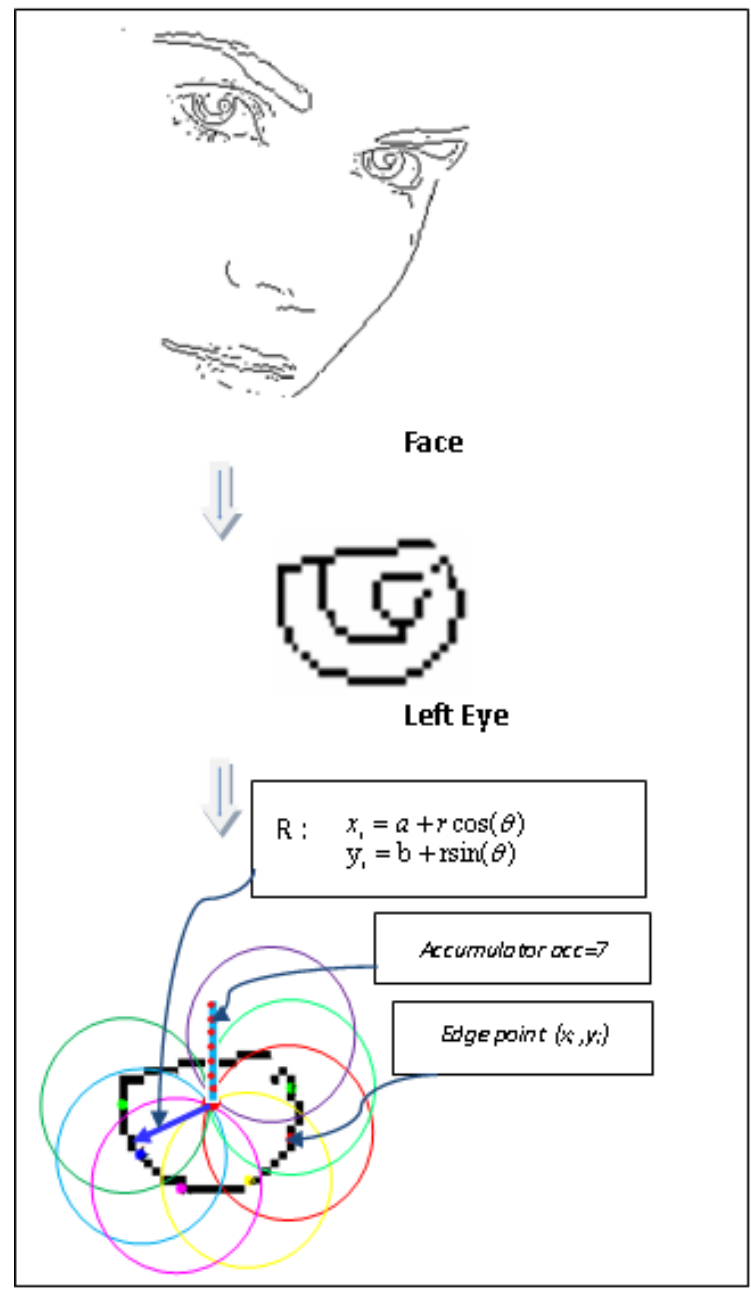

Figure 6. Accumulat or surrounding left iris

Therefore, the result will be more circles where their intersection with contour corresponds to set of points. The intersection of several circles with a single point of contour represents a point the accumulator. For the position of the circle to look for, we will seek the maximum of the accumulator.

We note that the accumulator $(\mathrm{h})$ presents two maximums indicate the most intersection points of circles in with contour. Their contact centers indicate irises centers.
The maximums resulting from the accumulator is a matrix of size $(1 \times 2 \times N)$ for both variables $\mathrm{X}_{0}$ and $\mathrm{Y}_{0}$. Where, $\mathrm{N}$ is the number of radius to research.

We can explain this principle as described in figure 6; the example shows that the Hough transform applied on the left eye image demonstrates the intersection of seven circles corresponding to the solution (radius) of accumu lator.

\section{Simulation Results}

We tested our algorithm on the "Caltech database" available on the Web[9], with 396 individuals with a number of 24 frames per individual for different poses. The images are: 24 bit - RGB, 576 x 768 pixels, file format: jpg. The proposed algorithm is implemented in MATLAB7.0; on Intel processor with 712MB RAM $2 \mathrm{GHz}$.

\subsection{Results of Iris Detection}

The experimental results have shown that the proposed algorithm gives better results. The algorithm accurately extracted irises of 379 images out of 396 images giving a success rate of $95 \%$. Fig 7 represents the better results of iris detection. In our experiments, the range of radius variation is from 6 to 11 pixels. This interval depends of a lot of parameters like: size of images, age...etc.
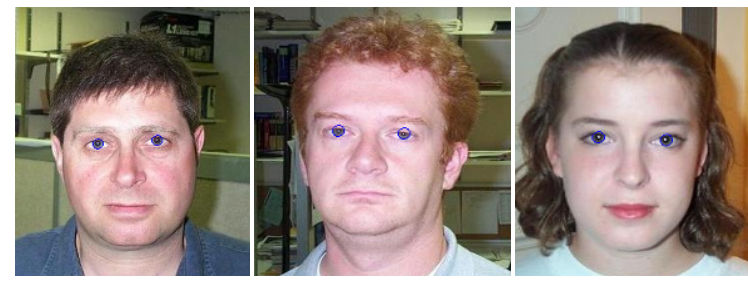

Figure 7. Good detection of iris

Using the Hough transform for circles, we find peaks in accumulator; we find also spatially separated circles. We save the coordinates of integer points close to a circle of radius $\mathrm{R}$ in an accumulator matrix. The plot of accumulator in $3 \mathrm{D}$ demonstrates the iris position in the initial image (see Fig 8).

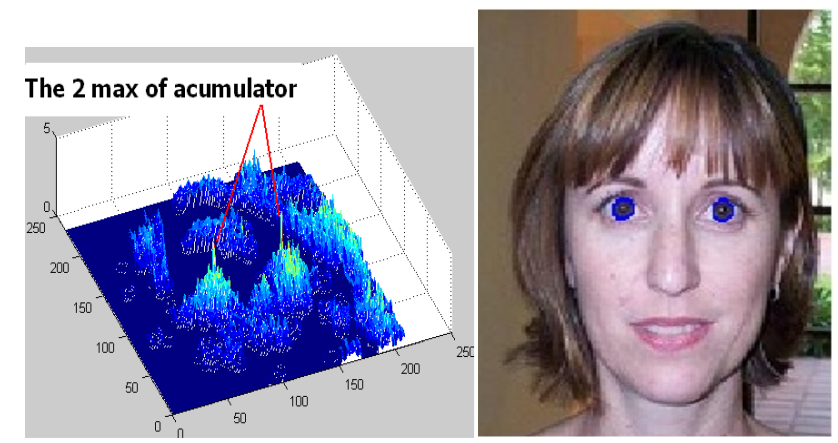

Figure 8. Top left : Accumulat or in 3D, top right localization of iris from the two maximums

Table 1 shows the coordinates of the two circles found in image. 
Table 1. Coordinates of the two circles

\begin{tabular}{|c|c|c|}
\hline Position and radius & Circle 1 & Circle 2 \\
\hline $\mathrm{X}_{0}$ & 134 & 73 \\
\hline $\mathrm{Y}_{0}$ & 115 & 114 \\
\hline $\mathrm{R}_{0}$ & 6 & 6 \\
\hline
\end{tabular}

The algorith $m$ demonstrates also results of false detection as mentioned in Fig 9.

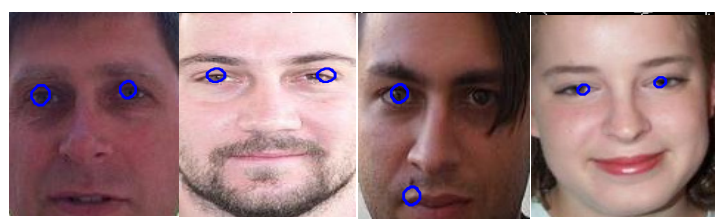

Figure 9. False iris detection

\subsection{Effect of Noise on the Iris Detection}

Our algorithm was tested on noisy images by adding Gaussian noise with zero mean $(\mathrm{m}=0)$ and variance $\sigma$. The density of probability is expressed by the equation:

$$
p(x, y)=\frac{1}{\sigma \sqrt{2 \pi}} e^{\left.-\frac{x^{2}+y^{2}}{2 \sigma^{2}}\right)}
$$

After adding noise to images, we obtained the following results:
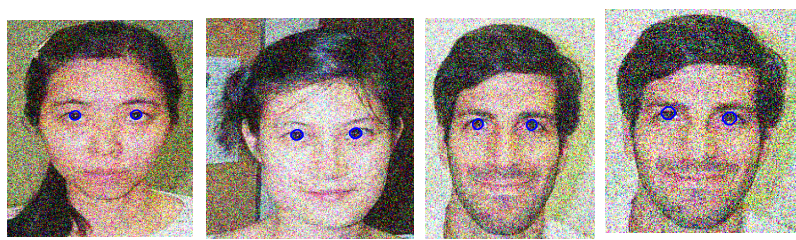

Figure 10. Iris detection of noisy images

Our experiments demonstrate bad detection results for a threshold grater than 0.1 .

Our algorithm was also tested on a part of the face image like eyes and other shapes. For example, the eye region can be extracted using a manual detection. The Hough transform permits us to detect correctly the iris for the most number of images (fig 11).
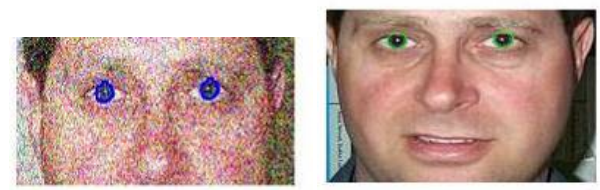

Figure 11. Iris detection for sub images (eyes, face and others)

\subsection{Application Of Iris Detection in Different Color Space}

$\sqrt{Y C b C r}$ Color Space:

The $\mathrm{YCbCr}$ color space was introduced in image coding for video. In the case of color images, it allows to compensate the errors in transmission of information. From the original image, we make a transformation from RGB to $\mathrm{YCbCr}$ space by applying the following rules[16] :

$$
\begin{aligned}
& \mathrm{Y}=0.299 \mathrm{R}+0.587 \mathrm{G}+0.114 \mathrm{~B} \\
& \mathrm{Cb}=0.564(\mathrm{~B}-\mathrm{Y})+128 \\
& \mathrm{Cr}=0.713(\mathrm{R}-\mathrm{Y})+128
\end{aligned}
$$

After conversion of $\mathrm{RGB}$ color to $\mathrm{YCbCr}$ color, we applied our algorith $\mathrm{m}$ on different images. We obtained the results listed below.

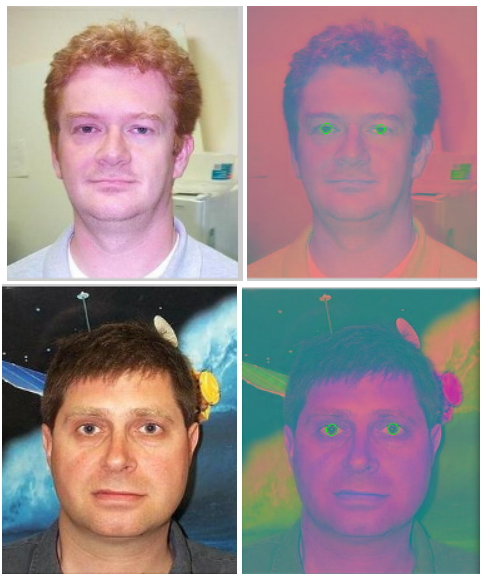

Figure 12. Examples of iris detection in $\mathrm{YCbCr}$ color

The results of $\mathrm{YCbCr}$ color application gives $66.1 \%$ detection of the two irises, $29.5 \%$ detection of a single iris and $4.3 \%$ of undetected iris .

Bad results are obtained; this is due to the detection of circular objects on clothing or on the back plan situated behind the people. See below some examples presented bad detection.
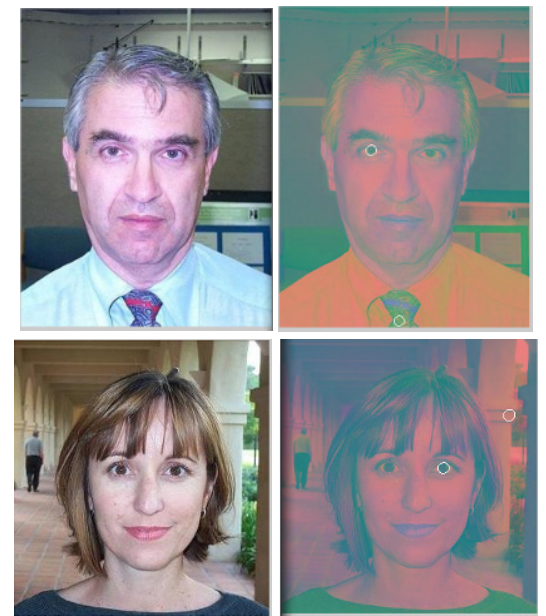

Figure 13. Bad iris detection in $\mathrm{YCbCr}$ color

\section{$\sqrt{ }$ YIQ Color Space}

The YIQ color space was explicitly designed to support color television, while allowing backwards compatibility with black and white TVs. It is still used today in non-HDTV color television broadcasting in the United States[17].

Conversion from RGBA to YIQA can be done using the color matrix[17]: 


$$
\left(\begin{array}{l}
Y \\
I \\
Q
\end{array}\right)=\left(\begin{array}{ccc}
0.299 & 0.587 & 0.114 \\
0.596 & -0.275 & -0.321 \\
0.212 & -0.523 & 0.311
\end{array}\right)\left(\begin{array}{l}
R \\
G \\
B
\end{array}\right)
$$

The application of YIQ Color Space gives 70.4\% detection of the two irises, $24.3 \%$ detection of a single iris and $5.2 \%$ false detection (no iris detection).

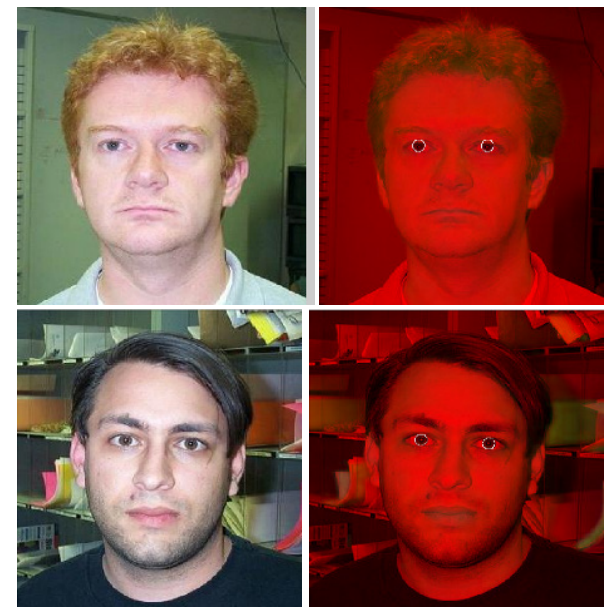

Figure 14. Examples of iris detection in YIQ color

The next figure represents bad results obtained by application of our algorith $\mathrm{m}$ with YIQ color space.

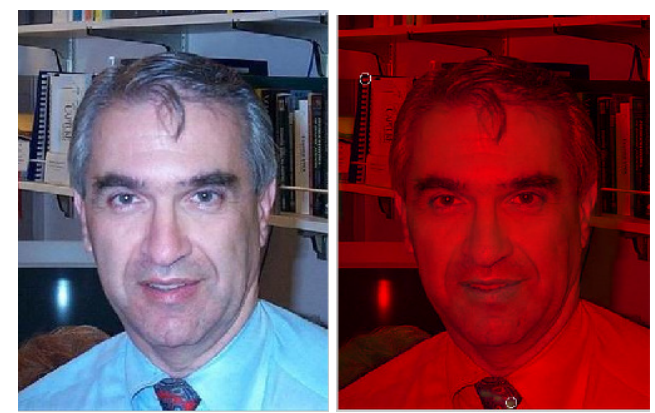

Figure 15. Bad iris detection in YIQ color space

\section{Conclusions}

We've presented in this article the circular hough transform for iris detection. Our method consists to search circles in contours image. The detection of contours plays a very important role in the detection of iris. The algorithm was applied to color image. The results depend on the influence of many parameters such as: The conditions for image capture are a very important factors for good or bad detection for example the lighting and the position of the human (position of the iris on face). We note that the position of the iris plays a very important role in the detection results. A recognition rate about $95 \%$ was obtained. The hough transform achieves also good results in $\mathrm{HSV}$ and $\mathrm{YCbCr}$ space. Bad results were obtained for noised images when the variance of Gaussian noise exceeds the value 0.1 .

Our method can be tested in several applications such as biometric systems, human machines interfaces, person identification, etc.

\section{REFERENCES}

[1] R. Bolle and S. Pankanti, eds, A. Jain, 'Biometrics: Personal Identification in a Networked Society”, Kluwer, 1999.

[2] Rajesh. Bodade and Dr. S. Talbar, "Dynamic Iris Localisation: A Novel Approach suitable for Fake Iris Detection", IEEE Conferences, 9781-4244-3941-6/09.

[3] Rajesh Bodade and Sanjay Talba, 'Novel approach of accurate iris localisation form high resolution eye images suitable for fake iris detection", International Journal of Information Technology and Knowledge Management, , Volume 3, No. 2, pp. 685-690, July-December 2010.

[4] R. Wildes, "Iris Recognition: An Emerging Biometric Technology”, Proc. IEEE, vol. 85, pp. 1348-1363, 1997.

[5] J. Daugman, "How iris recognition works", IEEE Transactionson Circuits and Systems for Video Technology, vol. 14, pp. 21-30,January 2004.

[6] Rahib H. Abiyev and Koray Altunkaya, ' Iris Recognition for Biometric Personal Identification Using Neural Networks,', J. Marques de Sá et al. (Eds.): ICANN , Part II, LNCS 4669, pp. 554-563, 2007.Springer-Verlag Ber lin Heid elberg 2007.

[7] Masek, L, 'Recognition of Human Iris Patterns for Biometric Identification', School of Computer Science and Soft Engineering, The University of Western Australia (2003).

[8] Wildes, R., Asmuth, J., Green, G., Hsu, S., Kolczynski, R., Matey, J., McBride, S.: 'A Machine-Vision System for Iris Recognition', Machine Vision and Applications 9, 1-8 (1996).

[9] Fei-Fei, R. Fergus, and P. Perona. ' The Caltech-101 Object Categories". California Institute of Technology, September, 2003.

[10] H. Rhody Chester F. 'Hough Circle Transform', Carlson Center for Imaging Science Rochester Institute of Technology October 11, 2005.

[11] A. Bouridane, "Recent Advances in Iris Recognition: A Multiscale Approach", Imaging for Forensics and Security, Signals and Communication Technology, DOI 10.1007/978-0-387-09532-5 4, Springer Science+Business Media, LLC 2009.

[12] R. Wildes, "Iris recognition: an emerging biometric technology", Proceedings of the IEEE, vol. 85, pp. 1348-1363, 1997.

[13] W. Kong and D. Zhang, "Accurate iris segmentation based on novel reflection and ey elash detection model", Proceedings of 2001 International Symposium on Intelligent Multi-media, Video and Speech Processing, Hong Kong, 2001.

[14] C. Tisse, L. Martin, L. Torres and M. Robert, "Person identification technique using human iris recognition", Proceedings of the Vision Interface, pp. 294-299, 2002.

[15] L. Ma, Y. Wang and T. Tan, "Iris Recognition Using Circular 
Symmetric Filters", National Laboratory of Pattern Recognition, Institute of Automation, Chinese Academy of Sciences, Beijing, 2002.

[16] R. Belaroussi, "localisation du visage dans des images et sequences video couleur', Thesis of doctorate of the University Pierre et Marie Curie, Décember 2006.

[17] T. Mcreynolds and D. Blythe, 'Advanced Graphics Programming Using OpenGL", Elsevier Inc, ISBN: 1-55860-659-9, 2005.
[18] Wojciech Wojcikiewicz, "Hough Transform, Line Detection in Robot Soccer", Coursework for Image Processing. 14th March 2008.

[19] J. Borovi_cka, 'Circle Detection Using Hough Transforms Documentation". COMS30121 - Image Processing and Computer Vision, 2003.

[20] D. Young, Straight lines and circles in the log-polar image. Cognitive Sciences Research Paper CSRP 522 July 2000. 